\title{
Liberia: Statistical Appendix
}

This Statistical Appendix paper for Liberia was prepared by a staff team of the International Monetary Fund as background documentation for the periodic consultation with the member country. It is based on the information available at the time it was completed on February 11, 2002. The views expressed in this document are those of the staff team and do not necessarily reflect the views of the government of Liberia or the Executive Board of the IMF.

The policy of publication of staff reports and other documents by the IMF allows for the deletion of market-sensitive information.

To assist the IMF in evaluating the publication policy, reader comments are invited and may be sent by e-mail to Publicationpolicy@imf.org.

Copies of this report are available to the public from International Monetary Fund - Publication Services $70019^{\text {th }}$ Street, N.W. $\bullet$ Washington, D.C. 20431

Telephone: (202) 623-7430 - Telefax: (202) 623-7201

E-mail: publications@imf.org Internet: http://www.imf.org

Price: $\$ 15.00$ a copy

\section{International Monetary Fund Washington, D.C.}




\title{
INTERNATIONAL MONETARY FUND
}

\section{LIBERIA}

\author{
Statistical Appendix \\ Prepared by a staff team consisting of Kenneth Meyers (head), \\ Markus Haacker (both AFR), Peter Fallon (PDR), and Ashok Bhatia (EP, TRE) \\ Approved by the African Department
}

February 11,2002

Contents

Page

Tables

1. Sectoral Gross Domestic Product, 1988 and 1997-2001 ............................................2

2. Sectoral Origin of GDP at Current Prices, 1998 and 1997-2001 ….............................

3. Sectoral Origin of GDP at 1992 Constant Prices, 1988 and 1997-2001 .......................

4. Selected Annual Output Indicators by Volume, 1988 and 1997-2001 .........................5

5. Selected Monthly Output Indicators by Volume, January 1999-September 2001 ........6

6. Selected Monthly Output Indicators by Value, January 1999-September 2001 .............7

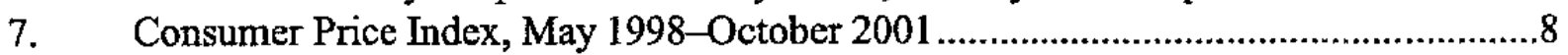

8. Central Government Revenue, 1997-2001 .............................................................9

9. Economic Classification of Central Government Expenditure, 1997-2001 ...............10

10. Summary Accounts of the Central Bank of Liberia, December 1998-October 2001 ................................................................................1

11. Summary Accounts of Deposit Money Banks, December 1998-October 2001 ..........................................................................12

12. Major Exports, 1987-88 and 1999-2001 ……....................................................13

13. Merchandise Trade - Imports by Category, 1987-88 and 1999-2001 .....................14

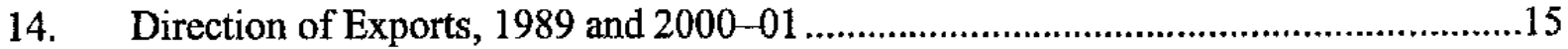

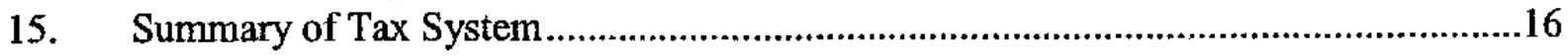


Table 1. Liberia: Sectoral Gross Domestic Product, 1988, and 1997-2001

\begin{tabular}{|c|c|c|c|c|c|c|}
\hline & 1988 & 1997 & 1998 & 1999 & $\begin{array}{c}2000 \\
\text { Est. }\end{array}$ & $\begin{array}{r}2001 \\
\text { Est. }\end{array}$ \\
\hline & \multicolumn{6}{|c|}{ (Annual percentage change, unless otherwise indicated) } \\
\hline GDP at 1992 constant prices & -1.9 & 100.0 & 29.7 & 22.9 & 20.4 & 5.3 \\
\hline Agriculture and fisheries & -15.6 & 69.5 & 22.3 & 20.0 & 6.2 & 7.9 \\
\hline Forestry & 6.7 & 69.8 & 70.2 & 19.1 & 70.6 & 0.7 \\
\hline Mining and parning & 4.2 & 96.1 & 49.0 & 10.6 & 49.8 & -63.9 \\
\hline Mamufacturing & 4.2 & 994.5 & 5.1 & 21.7 & 15.3 & 14.4 \\
\hline \multirow[t]{2}{*}{ Services } & 4.2 & 390.9 & 29.9 & 39.2 & 14.7 & 3.6 \\
\hline & \multicolumn{6}{|c|}{ (Percentage of prewar level, $1987=100$ ) } \\
\hline GDP at 1992 constant prices & 98.1 & 21.7 & 28.2 & 34.6 & 41.7 & 43.9 \\
\hline Agriculture and fisheries & 84.4 & 43.6 & 53.3 & 64.0 & 67.9 & 73.3 \\
\hline Forestty & 106.7 & 66.3 & 112.9 & 134.4 & 229.3 & 230.9 \\
\hline Mining and panning & 104.2 & 0.5 & 0.8 & 0.9 & 1.3 & 0.5 \\
\hline Manufacturing & 104.2 & 16.8 & 17.7 & 21.5 & 24.8 & 28.3 \\
\hline \multirow[t]{2}{*}{ Services } & 104.2 & 7.5 & 9.8 & 13.6 & 15.6 & 16.2 \\
\hline & \multicolumn{6}{|c|}{ (Millions of U.S. dollars) } \\
\hline GDP at current prices & $1,061.2$ & 289.2 & 359.6 & 441.8 & 525.6 & 522.9 \\
\hline Agriculture and fisheries & 344.5 & 182.3 & 229.4 & 277.0 & 278.4 & 274.7 \\
\hline Forestry & 51.2 & 45.4 & 53.4 & 59.6 & 117.1 & 113.5 \\
\hline Mining and panning & 112.0 & 0.7 & 1.1 & 1.2 & 1.0 & 0.3 \\
\hline Manufacturing & 78.0 & 16.3 & 17.3 & 21.4 & 26.9 & 34.1 \\
\hline \multirow[t]{2}{*}{ Services } & 475.5 & 44.6 & 58.5 & 82.6 & 102.1 & 100.4 \\
\hline & \multicolumn{6}{|c|}{ (Percentage shares) } \\
\hline GDP at current prices & 100.0 & 100.0 & 100.0 & 100.0 & 100.0 & 100.0 \\
\hline Agriculture and insheries & 32.5 & 63.0 & 63.8 & 62.7 & 53.0 & 52.5 \\
\hline Forestry & 4.8 & 15.7 & 14.8 & 13.5 & 22.3 & 21.7 \\
\hline Mining and panning & 10.6 & 0.2 & 0.3 & 0.3 & 0.2 & 0.1 \\
\hline Manufacturing & 7.3 & 5.6 & 4.8 & 4.8 & 5.1 & 6.5 \\
\hline Services & 44.8 & 15.4 & 16.3 & 18.7 & 19.4 & 19.2 \\
\hline \multicolumn{7}{|l|}{ Memorandum items: } \\
\hline & \multicolumn{6}{|c|}{ (Units indicated) } \\
\hline Real GDP per capita (1992 constant U.S. dollars) & 489.2 & 121.9 & 164.4 & 168.4 & 180.1 & 184.1 \\
\hline Nominal GDP pet capita (U.S. dollars) & 453.5 & 139.0 & 179.8 & 184.1 & 194.7 & 188.0 \\
\hline \multirow[t]{2}{*}{ Population (millions) } & 2.3 & 2.1 & 2.0 & 2.4 & 2.7 & 2.8 \\
\hline & \multicolumn{6}{|c|}{ (Annual percentage change) } \\
\hline GDP deflator & 8.8 & .9 .9 & -4.1 & 0.0 & -1.1 & -5.5 \\
\hline Consumer prices (period average) & 9.6 & $\cdots$ & $\cdots$ & 2.0 & 5.3 & 12.4 \\
\hline
\end{tabular}

Sources: Liberian authorities; and Fund staff estimates and projections. 
Table 2. Liberia: Sectoral Origin of GDP at Current Prices, 1988, and 1997-2001

(in millions of U.S. dollars)

\begin{tabular}{|c|c|c|c|c|c|c|}
\hline & 1988 & 1997 & 1998 & 1999 & 2000 & $\begin{array}{r}2001 \\
\text { Est. }\end{array}$ \\
\hline GDP at current prices & $1,061.2$ & 289.2 & 359.6 & 441.8 & 525.6 & 522.9 \\
\hline Agriculture and fisheries & 344.5 & 182.3 & 229.4 & 277.0 & 278.4 & 274.7 \\
\hline Rubber & 73.5 & 19.4 & 37.6 & 61.7 & 57.0 & 57.5 \\
\hline Coffee & 2.2 & 0.1 & 0.5 & 0.7 & 0.5 & 0.2 \\
\hline Cocoa & 11.4 & 0.7 & 1.6 & 2.0 & 0.7 & 0.3 \\
\hline Rice & 99.8 & 50.9 & 64.2 & 72.5 & 62.7 & 57.1 \\
\hline Cassava & 48.9 & 39.8 & 44.0 & 48.4 & 53.5 & 54.2 \\
\hline Other & 108.7 & 71.3 & 81.5 & 91.7 & 104.0 & 105.3 \\
\hline Forestry & 51.2 & 45.4 & 53.4 & 59.6 & 117.1 & 113.5 \\
\hline Logs and timber & 31.2 & 5.9 & 13.0 & 18.2 & 61.8 & 65.0 \\
\hline Charcoal and wood & 20.1 & 39.5 & 40.4 & 41.4 & 55.3 & 48.5 \\
\hline Mining and panning & 112.0 & 0.7 & 1.1 & 1.2 & 1.0 & 0.3 \\
\hline Iron ore & 104.2 & 0.0 & 0.0 & 0.0 & 0.0 & 0.0 \\
\hline Other & 7.8 & 0.7 & 1.1 & 1.2 & 1.0 & 0.3 \\
\hline Manufacturing & 78.0 & 16.3 & 17.3 & 21.4 & 26.9 & 34.1 \\
\hline Cement & 20.7 & 3.2 & 4.9 & 5.3 & 6.2 & 7.8 \\
\hline Beverages and beer & 47.1 & 10.7 & 10.9 & 13.3 & 17.4 & 22.6 \\
\hline Other & 10.2 & 2.4 & 1.5 & 2.8 & 3.2 & 3.7 \\
\hline Services & 475.5 & 44.6 & 58.5 & 82.6 & 102.1 & 100.4 \\
\hline Electricity and water & 12.4 & 1.2 & 1.5 & 2.3 & 3.0 & 3.1 \\
\hline Construction & 45.4 & 4.3 & 5.6 & 6.9 & 7.8 & 7.8 \\
\hline Trade, hotels, etc. & 89.6 & 8.4 & 11.0 & 17.0 & 21.1 & 23,0 \\
\hline Transportation and communication & 136.9 & 12.8 & 16.8 & 21.6 & 24.4 & 23.2 \\
\hline Financial institutions & 88.8 & 8.3 & 10.9 & 13.3 & 15.1 & 14.7 \\
\hline Government services & 50.4 & 4.7 & 6.2 & 11.2 & 16.9 & 14.1 \\
\hline Other services & 51.9 & 4.9 & 6.4 & 10.3 & 13.9 & 14.5 \\
\hline
\end{tabular}

Sources: Liberian authorities; and Fund staff estimates. 
Table 3. Liberia: Sectoral Origin of GDP at 1992 Constant Prices, 1988, and 1997-2001

(In millions of U.S. dollars)

\begin{tabular}{|c|c|c|c|c|c|c|}
\hline & 1988 & 1997 & 1998 & 1999 & 2000 & $\begin{array}{r}2001 \\
\text { Est. }\end{array}$ \\
\hline GDP at 1992 constant prices & $1,144.8$ & 253.5 & 328.7 & 404.1 & 486.3 & 512.1 \\
\hline Agriculture and fisheries & 311.2 & 160.8 & 196.6 & 236.0 & 250.5 & 270.4 \\
\hline Rubber & 53.2 & 16.4 & 30.3 & 41.7 & 35.4 & 39.4 \\
\hline Coffee & 1.0 & 0.1 & 0.3 & 0.4 & 0.5 & 0.5 \\
\hline Cocoa & 7.9 & 0.5 & 1.0 & 1.4 & 0.6 & 0.5 \\
\hline Rice & 96.5 & 45.2 & 55.9 & 65.6 & 69.4 & 74.6 \\
\hline Cassava & 47.3 & 35.3 & 38.3 & 43.8 & 49.1 & 52.8 \\
\hline Other & 105.2 & 63.3 & 70.9 & 83.0 & 95.5 & 102.7 \\
\hline Forestry & 60.4 & 37.6 & 63.9 & 76.1 & 129.8 & 130.7 \\
\hline Logs and timber & 36.8 & 4.9 & 15.5 & 23.8 & 66.3 & 66.7 \\
\hline Charcoal and wood & 23.7 & 32.7 & 48.4 & 52.3 & 63.6 & 64.0 \\
\hline Mining and panning & 130.2 & 0.7 & 1.0 & 1.1 & 1.6 & 0.6 \\
\hline Iron ore & 121.1 & 0.0 & 0.0 & 0.0 & 0.0 & 0.0 \\
\hline Other & 9.0 & 0.7 & 1.0 & 1.1 & 1.6 & 0.6 \\
\hline Manufacturing & 90.6 & 14.6 & 15.4 & 18.7 & 21.5 & 24.6 \\
\hline Cement & 24.0 & 2.9 & 4.3 & 4.6 & 5.0 & 6.1 \\
\hline Beverages and beer & 54.7 & 9.6 & 9.7 & 11.6 & 13.9 & 15.3 \\
\hline Other & 11.9 & 2.2 & 1.3 & 2.4 & 2.6 & 3.2 \\
\hline Services & 552.4 & 39.9 & 51.9 & 72.2 & 82.8 & 85.8 \\
\hline Electricity and water & 13.3 & 1.0 & 1.4 & 2.0 & 2.4 & 2.6 \\
\hline Construction & 48.6 & 3.8 & 4.9 & 6.0 & 6.3 & 6.6 \\
\hline Trade, hotels, etc. & 104.4 & 7.5 & 9.8 & 14.9 & 17.1 & 19.7 \\
\hline Transportation and comrounication & 164.1 & 11.5 & 14.9 & 18.9 & 19.8 & 19.8 \\
\hline Financial institutions & 103.9 & 7.5 & 9.7 & 11.6 & 12.2 & 12.6 \\
\hline Government services & 58.6 & 4.2 & 5.5 & 9.8 & 13.7 & 12.1 \\
\hline Other services & 59.7 & 4.4 & 5.7 & 9.0 & 11.3 & 12.4 \\
\hline
\end{tabular}

Sources: Liberian authorities; and Fund staff estimates. 
Table 4. Liberia: Selected Annual Output Indicators by Volume, 1987, and 1997-2001

(Units indicated)

\begin{tabular}{|c|c|c|c|c|c|c|}
\hline & 1988 & 1997 & 1998 & 1999 & 2000 & $\begin{array}{r}2001 \\
\text { Est. }\end{array}$ \\
\hline \multicolumn{7}{|l|}{ Agriculture and fisheries } \\
\hline Rubber (millions of pounds) & 253.1 & 37.0 & 55.6 & 138.0 & 242.5 & 269.6 \\
\hline Coffee (metric tons) & $4,116.0$ & 42.0 & 272.0 & 872.8 & 896.0 & 874.0 \\
\hline Cocoa (metric tons) & $2,946.0$ & 755.0 & $2,440.5$ & $2,591.0$ & $1,459.5$ & $1,201.2$ \\
\hline Rice (thousands of metric tons) & $\ldots$ & 101.5 & 108.0 & 114.4 & 120.9 & 130.0 \\
\hline Cassava (thousands of metric tons) & $\ldots$ & 295.0 & 313.3 & 356.7 & 400.0 & 430.0 \\
\hline \multicolumn{7}{|l|}{ Forestry } \\
\hline Logs and timber (cubic meters) & $\ldots$ & 75.0 & 1.57 .1 & 335.5 & 934.1 & 940.4 \\
\hline \multicolumn{7}{|l|}{ Mining and panning } \\
\hline Iron ore (millions of metric tons) & 13.5 & 0.0 & 0.0 & 0.0 & 0.0 & 0.0 \\
\hline
\end{tabular}

Sources: Liberian authorities; and Fund staff estimates. 
Table 5. Liberia: Selected Monthly Output Indicators by Valume, January 1999 - September 2001

\begin{tabular}{|c|c|c|c|c|c|c|c|c|c|c|c|c|c|}
\hline \multirow{3}{*}{$\begin{array}{l}\text { Sector } \\
\text { Commodity } \\
\text { (Unil of measure) }\end{array}$} & \multirow{2}{*}{\multicolumn{4}{|c|}{ Apriculture \& Fisheries }} & \multirow{2}{*}{\multicolumn{2}{|c|}{ Forestry }} & \multirow{2}{*}{\multicolumn{2}{|c|}{ Mining \& Panning }} & \multicolumn{5}{|c|}{ Maruffacturing } \\
\hline & & & & & & & & & \multirow[b]{2}{*}{$\begin{array}{l}\text { Cement } \\
\text { (MT) } 11\end{array}$} & \multicolumn{4}{|c|}{ Beverages \& Beer } \\
\hline & $\begin{array}{l}\text { Rubber } \\
\text { (MT) } 1 /\end{array}$ & $\begin{array}{l}\text { Cocou Bears } \\
\text { (MT) } 1 /\end{array}$ & $\begin{array}{r}\text { Coffee } \\
(\mathrm{MT}) \perp\end{array}$ & $\begin{array}{r}\text { Fish } \\
(\mathrm{Kg}) 2 /\end{array}$ & $\begin{array}{r}\text { Round Logs } \\
\text { (M3) } 3 /\end{array}$ & $\begin{array}{r}\text { Sawn Timber } \\
\text { (M3) } 3 \\
\end{array}$ & $\begin{array}{r}\text { Gold } \\
\text { (Ounces) }\end{array}$ & $\begin{array}{r}\text { Diarmonds } \\
\text { (Carats) } \\
\end{array}$ & & $\begin{array}{r}\text { Soft Drinks } \\
\text { (Litres) } \\
\end{array}$ & $\begin{array}{r}\text { Spirits } \\
\text { (Litres) }\end{array}$ & $\begin{array}{r}\text { Betr } \\
\text { (Litres) }\end{array}$ & $\begin{array}{r}\text { Stout } \\
\text { (Litres) }\end{array}$ \\
\hline 1999 & 62,705 & 2,591 & $\ldots$ & 480,600 & $\ldots$ & ... & 550 & $\ldots$ & 50,735 & 272,805 & 207,133 & $3,113,463$ & $1,511,425$ \\
\hline Jasuarry & 5,594 & $s 22$ & ... & 25,200 & 8,850 & $\ldots$ & 0 & $\ldots$ & 4,550 & $10,05\}$ & 5,062 & 110,195 & 102,311 \\
\hline February & 968 & 388 & $\ldots$ & 29,720 & 19,949 & $\ldots$ & 0 & 949 & 4,752 & 22,517 & 4,828 & 351,015 & 152,741 \\
\hline March & 6,154 & 102 & 225 & 19,960 & 9,895 & $\ldots$ & 49 & 507 & 1,835 & $50,63 \mathrm{~B}$ & 4,761 & 420,098 & 203,172 \\
\hline April & 5,848 & ... & 204 & 45,020 & 23,855 & ... & 122 & 1,545 & 4,837 & 35,449 & 4,780 & 459,837 & 191,506 \\
\hline May & 6,237 & $\ldots$ & 187 & 36,060 & 11,800 & $\ldots$ & 54 & 317 & 5,283 & 37,753 & 4,476 & 406,009 & 87,690 \\
\hline June & 5,648 & 44 & 85 & 55,840 & 26,087 & ... & 65 & 1,393 & 4,873 & 39,288 & 56,930 & 1,520 & 110,532 \\
\hline July & 4,805 & $\ldots$ & ... & 29,360 & 2,512 & $\ldots$ & 65 & 1,606 & 3,866 & 42,600 & 5,899 & 1,060 & 78,820 \\
\hline August & 4,435 & 29 & 51 & 33,820 & 7,199 & $\ldots$ & 29 & 398 & 4,344 & 34,509 & 31,415 & 399,883 & 119,544 \\
\hline Stptember & 6,409 & $\ldots$ & $\ldots$ & 31,620 & 19,983 & $\ldots$ & 60 & 653 & 4,088 & 0 & 18,657 & 102,836 & 100,861 \\
\hline Ostober & 5,232 & 551 & $\ldots$ & 5,420 & $\ldots$ & $\ldots$ & 62 & 245 & 4,644 & 0 & 25,036 & 406,009 & i 13,240 \\
\hline Novernber & $5,65]$ & $\ldots$ & $\ldots$ & 37,100 & 3,170 & $\ldots$ & 44 & 835 & 4,321 & 0 & 21,847 & 132,991 & 100,552 \\
\hline December & 5,724 & 955 & 26 & 53,480 & 9,745 & $\ldots$ & 0 & 280 & 3,342 & 0 & 23,442 & 321,950 & 150,456 \\
\hline 2000 & 110,235 & 1,203 & 896 & 433,560 & 334,066 & 224,824 & 701 & 22,220 & 54,933 & $10,940,674$ & 151,442 & $2,942,871$ & $1,279,548$ \\
\hline Jamuary & 5,404 & 160 & 34 & 88,260 & 78,926 & 26,499 & 60 & 115 & 1,561 & $1,000,505$ & 9,081 & 159,174 & 99,032 \\
\hline Febnary & 5,417 & 218 & 13 & 42,780 & 132,700 & 53,120 & 0 & 629 & 5,829 & 881,446 & 6,723 & 224,764 & 102,920 \\
\hline March & 5,796 & 725 & 0 & 35,760 & 128,080 & 26,991 & 28 & 1,433 & 6,643 & $1,201,550$ & 9,477 & 233,446 & 98,723 \\
\hline April & 9,305 & 0 & 36 & 23,220 & 126,974 & 37,096 & 127 & 1,039 & 5,594 & $1,087,704$ & 14,450 & 311,290 & 104,671 \\
\hline May & 11,159 & 0 & 0 & 38,080 & 111,732 & 53,155 & 179 & 2,257 & 1,723 & $1,097,698$ & 10,157 & 201,394 & 98,042 \\
\hline June & 2,195 & 0 & 0 & 43,400 & 100,841 & 23,499 & 0 & 2,043 & 7,320 & 846,749 & 16,173 & 246,249 & 99,071 \\
\hline July & 19,706 & 0 & 13 & 26,400 & 68,275 & 0 & 88 & 2,525 & 3,957 & 709,178 & 8,510 & 207,676 & 98,865 \\
\hline August & 2,616 & 0 & 0 & 47,400 & 35,664 & 0 & 0 & 2,275 & 3,876 & 787,550 & 8,132 & 249,472 & 305,890 \\
\hline September & 9,363 & 0 & 800 & 24,480 & 20,848 & 1,018 & 0 & 4,548 & 4,246 & $6 \mathrm{BO} 0,969$ & 16,304 & 204,934 & 105,669 \\
\hline October & 4,063 & 0 & 0 & 23,040 & 15,509 & 1,354 & 0 & 771 & 4,262 & 680,947 & 18,329 & 186,842 & 99,087 \\
\hline Noventber & 18,804 & 0 & 0 & 22.740 & 43,664 & 630 & 0 & 2,634 & 4,973 & 799,942 & 15,215 & 347,139 & 103,190 \\
\hline December & 16,407 & 100 & 0 & 18,000 & 70,853 & 1,462 & 219 & 1,951 & 4,949 & $1,166,436$ & 18,891 & 370,491 & 164,388 \\
\hline 2001 & 78,878 & 281 & 437 & 220,220 & 788,541 & 13,059 & $1, \mathbf{4 3 1}$ & $3,8 \mathrm{sA5}$ & 49,450 & $5,161,594$ & 200,129 & $2,143,706$ & 955,072 \\
\hline January & 11,603 & 81 & 126 & 26,540 & 74,757 & 966 & 161 & 2,780 & 5,720 & 914,047 & 27,945 & 199,848 & 97,955 \\
\hline February & 8,408 & 6.5 & 101 & 18,700 & 99,680 & 960 & 323 & 388 & 5,600 & 734,256 & 28,391 & 354,179 & 123,668 \\
\hline March & 7,556 & 48 & 75 & 22,300 & 138,648 & 560 & 516 & 717 & 7,200 & 972,194 & 49,603 & 288,698 & 111,918 \\
\hline April & 11,223 & 32 & 50 & 5,700 & 141,294 & 2,616 & 64 & $\ldots$ & 6,600 & 818,194 & 23,269 & 255,608 & 103,332 \\
\hline May & 9,233 & 29 & 45 & 21,620 & 103,205 & 1,573 & 164 & ... & 6,350 & 102,290 & 17,032 & 220,310 & 299,714 \\
\hline Jume & 10,219 & 26 & 40 & 14,480 & 99,757 & $1,98 B$ & 137 & $\ldots$ & 5,120 & 562,183 & $16,53 \mathrm{~B}$ & 249,843 & 102,216 \\
\hline July & $8, \mathfrak{3} 28$ & $\ldots$ & $\ldots$ & 59,180 & 72,558 & 2,728 & 0 & $\ldots$ & 4,630 & 514,606 & 16,110 & 365,498 & 105,645 \\
\hline August & 11,271 & $\ldots$ & $\ldots$ & 51,700 & 58,642 & 1,668 & 0 & $\ldots$ & 4,350 & 308,938 & 21,236 & 209,722 & 100,624 \\
\hline Septernber & 1,037 & $\ldots$ & $\ldots$ & $\ldots$ & $\ldots$ & $\ldots$ & 66 & $\ldots$ & 3,880 & 234,886 & .. & $\ldots$ & $\ldots$ \\
\hline
\end{tabular}

Source: Liberian authorities.

$1 / \mathrm{MT}=$ metric tons.

2/ $/ \mathrm{K}_{\mathrm{g}}=$ kiulgrazis.

$3 / \mathrm{M3}=$ cubic meters. 
Table 6. Liberia: Selected Monthly Output Indicators by Vahue, January 1999 - September 2001

\begin{tabular}{|c|c|c|c|c|c|c|c|c|c|c|c|c|c|}
\hline \multirow{3}{*}{$\begin{array}{l}\text { Sector } \\
\text { Commodity }\end{array}$} & \multirow{2}{*}{\multicolumn{4}{|c|}{ Agiculture \& Fisberies }} & \multirow{2}{*}{\multicolumn{2}{|c|}{ Forestry }} & \multirow{2}{*}{\multicolumn{2}{|c|}{ Mining \& Pamming }} & \multicolumn{5}{|c|}{ Manufacturing } \\
\hline & & & & & & & & & \multirow[b]{2}{*}{ Cernent } & \multicolumn{4}{|c|}{ Bevraages \& Beer } \\
\hline & Rubber & Cocoa Beans & Coffer & Fish & Round Logs & Sawn Timber & Gold & Diamonds & & Soft Drinks & Spirits & Beer & Stout \\
\hline \multicolumn{14}{|c|}{ (Thousands of Libetian dollars) } \\
\hline 1999 & ... & ... & .. & 4,939 & 698,727 & $\ldots$ & 3,947 & ... & 210,395 & 136,175 & ... & 137,328 & 107,885 \\
\hline January & $\ldots$ & $\ldots$ & $\ldots$ & 252 & 46,304 & ... & 0 & ... & 537 & 0 & $\ldots$ & 10,623 & 7,629 \\
\hline February & ... & $\ldots$ & $\ldots$ & 281 & 121,168 & ... & 0 & 3,634 & 22,187 & 10,996 & $\ldots$ & 34,900 & 11,629 \\
\hline March & $\ldots$ & $\ldots$ & $\ldots$ & 265 & 60,162 & $\ldots$ & 237 & 3,820 & 9,669 & 24,469 & $\ldots$ & 12,830 & 9,242 \\
\hline April & ... & $\ldots$ & $\ldots$ & 200 & 108,796 & $\ldots$ & 1,008 & 8,627 & 21,476 & 19,497 & ... & 11,444 & 8,002 \\
\hline May & $\ldots$ & $\ldots$ & $\ldots$ & 540 & 60,494 & $\ldots$ & 395 & 560 & 22,840 & 38,984 & ... & 12,051 & 8,622 \\
\hline Jurle & ... & $\ldots$ & $\ldots$ & 682 & 95,540 & $\ldots$ & 517 & 6,877 & 21,836 & 21,609 & ... & 11,161 & 8,415 \\
\hline July & $\ldots$ & $\ldots$ & $\ldots$ & 573 & 12,979 & $\ldots$ & 439 & 7,349 & 17,530 & 21,640 & $\ldots$ & 11,875 & 11,875 \\
\hline August & $\ldots$ & $\ldots$ & $\ldots$ & 609 & 32,701 & $\ldots$ & 192 & 2,810 & 20,163 & 18,980 & $\ldots$ & 8,478 & 7,795 \\
\hline Septernber & $\ldots$ & $\ldots$ & $\ldots$ & 503 & 104,227 & $\ldots$ & 447 & 6,064 & 17,865 & 0 & $\ldots$ & 8,017 & 6,944 \\
\hline October & $\ldots$ & $\ldots$ & $\ldots$ & 393 & $\ldots$ & $\ldots$ & 340 & 739 & 21,308 & 0 & $\ldots$ & 12,049 & 8,622 \\
\hline November & $\ldots$ & $\ldots$ & $\ldots$ & 370 & 15,461 & $\ldots$ & 370 & 963 & 19,734 & 0 & $\ldots$ & 9,412 & 7,656 \\
\hline December & $\ldots$ & $\ldots$ & $\ldots$ & 270 & 40,894 & $\ldots$ & 0 & 533 & $15,25]$ & 0 & $\ldots$ & 14,488 & 11,455 \\
\hline 2000 & $2,322,612$ & 29,032 & 21,226 & 9,621 & $2,548,212$ & $\ldots$ & 5,299 & 37,053 & 256,968 & 245,758 & 12,095 & 134,785 & 98,033 \\
\hline January & 121,341 & $1,78\}$ & 706 & 2,207 & 138,632 & ... & 512 & 700 & 1,200 & 23,931 & 940 & 8,791 & 6,960 \\
\hline February & 345,391 & 2,385 & 269 & 1,070 & 174,598 & $\ldots$ & 0 & 1,630 & 25,675 & 20,508 & 927 & 10,629 & 7,398 \\
\hline March & 159,139 & 19,366 & 0 & 894 & 365,030 & $\ldots$ & 248 & 3,862 & 34,283 & 25,332 & 615 & 11,588 & 8,208 \\
\hline April & 224,487 & 0 & 930 & 455 & 275,963 & $\ldots$ & 867 & 3,157 & 27,921 & 25,474 & 812 & 10,554 & 7,568 \\
\hline May & 267,933 & 0 & 0 & 793 & 115,575 & ... & 1,469 & 5,379 & 12,015 & 24,643 & 685 & 10,606 & 7,638 \\
\hline June & 44,604 & 0 & 0 & 928 & 277,442 & $\ldots$ & 0 & 3,476 & 32,068 & 17,012 & 1,334 & 8,838 & 7,340 \\
\hline July & 427,549 & 0 & 241 & 437 & 300,526 & $\ldots$ & 614 & 3,586 & 18,252 & 18,609 & 588 & 10,695 & 9,170 \\
\hline August & 99,372 & 0 & 0 & 873 & 407,688 & $\ldots$ & 0 & 6,082 & 17,854 & 15,993 & 651 & 11,518 & 8,062 \\
\hline September & 212,845 & 0 & [9,08] & 371 & 64,432 & $\ldots$ & 0 & 4,201 & 21,167 & 14,735 & 1,319 & 10,079 & 8,045 \\
\hline October & 72,778 & 0 & 0 & 589 & 160,814 & $\ldots$ & 0 & 1,072 & 19,893 & 17,471 & 1,239 & 8,668 & 7,271 \\
\hline Novernber & 385,989 & 0 & 0 & 566 & 34,848 & $\ldots$ & 0 & 1,953 & 22,343 & 18,164 & 1,245 & 15,706 & 7,856 \\
\hline Desernber & 161,385 & 5,500 & 0 & 439 & 232,664 & $\ldots$ & 1,590 & 1,956 & 24,297 & 23,885 & 1,739 & 17,112 & 12,516 \\
\hline \multicolumn{14}{|l|}{2001} \\
\hline January & 289,572 & 1,265 & 1,100 & 466 & 199,389 & $\ldots$ & 1,272 & 2,845 & 31,745 & 21,272 & $\begin{array}{l}2,009 \\
3\end{array}$ & $\begin{array}{r}9,860 \\
6,452\end{array}$ & $\begin{array}{l}7,458 \\
7,541\end{array}$ \\
\hline Febrary & 210,840 & 989 & 858 & 410 & 562,207 & 4,822 & 2,359 & 883 & 30,259 & 17,837 & 2,380 & $\begin{array}{l}16,452 \\
14,640\end{array}$ & $\begin{array}{l}7,541 \\
8,521\end{array}$ \\
\hline March & 168,782 & 746 & 651 & 473 & 268,589 & 10,641 & 4,054 & 1,869 & 39,737 & 22,055 & 4,074 & $\begin{array}{l}{[4,640} \\
{[3,233}\end{array}$ & $\begin{array}{l}8,521 \\
7,035\end{array}$ \\
\hline April & 241,110 & 514 & 449 & 143 & 532,798 & 1,127 & 628 & $\ldots$ & 37,684 & 18,873 & $\begin{array}{r}1,836 \\
1390\end{array}$ & $\begin{array}{l}13,233 \\
15,965\end{array}$ & $\begin{array}{l}7,035 \\
8,266\end{array}$ \\
\hline May & 232,654 & 485 & 421 & 420 & 321,798 & 48,343 & 1,538 & $\ldots$ & 37,756 & 15,134 & $\begin{array}{l}1,329 \\
1,281\end{array}$ & 16,338 & $\begin{array}{l}8,266 \\
9,349\end{array}$ \\
\hline Junte & 311,104 & 477 & 410 & 217 & 281,008 & 24,555 & 1,368 & $\ldots$ & 33,352 & 35,617 & $\begin{array}{l}1,281 \\
1,321\end{array}$ & 19,744 & $\begin{array}{r}8,349 \\
11,734\end{array}$ \\
\hline July & 207,286 & $\ldots$ & $\ldots$ & 1,113 & 185,847 & 858,256 & 0 & $\ldots$ & 32,746 & 17,150 & $\begin{array}{l}1,321 \\
1,385\end{array}$ & 6,072 & $\begin{array}{r}1, \sqrt{144} \\
5,452\end{array}$ \\
\hline August & 292,138 & $\ldots$ & $\cdots$ & 1,094 & $\mathbf{1 6 5 , 1 2 7}$ & 51,258 & 0 & $\ldots$ & 27,555 & 10,329 & 1,385 & 0,012 & 5,452 \\
\hline September & 24,378 & $\ldots$ & ... & $\ldots$ & 293,293 & $\ldots$ & 602 & $\ldots$ & 23,810 & 10,308 & ... & $\cdots$ & ... \\
\hline
\end{tabular}

Sourex: Liberian authoritics. 
Table 7. Liberia: Consumer Price Index, May 1998 - October 2001

\begin{tabular}{|c|c|c|c|c|c|c|c|c|c|c|}
\hline & Overal & Index & Food & Drinks \& & Fuel \& & Clothing & Household & Personal & Rent & Misc. \\
\hline (Weight) & $\begin{array}{l}\text { hndex } \\
\text { (joolo) }\end{array}$ & $\begin{array}{l}\text { Annual } \\
\% \text { change }\end{array}$ & $(3, S)$ & Tobacco & $\begin{array}{l}\text { Light } \\
\text { (5.9) }\end{array}$ & $(13.8)$ & $\begin{array}{r}\text { Goods \& } \\
\text { Furniture } \\
(6 . j)\end{array}$ & $\begin{array}{r}\text { Care \& } \\
\text { Services } \\
\text { (11 } .4)\end{array}$ & $(14.9)$ & $\{8.7\}$ \\
\hline
\end{tabular}

(May $1998=100)$

\begin{tabular}{|c|c|c|c|c|c|c|c|c|c|c|}
\hline \multicolumn{11}{|l|}{1998} \\
\hline May & 100.0 & $\cdots$ & 100.0 & 100.0 & 100.0 & 100.0 & 100.0 & 100.0 & 100.0 & 100.0 \\
\hline Fune & 102.6 & $\ldots$ & 106.5 & 100.0 & 100.0 & 100.5 & 104.3 & 100.0 & 100.0 & 100.2 \\
\hline July & 112.4 & $\cdots$ & 131.7 & 100.0 & 94.0 & 108.4 & 109.5 & 100.0 & 100.0 & 100.2 \\
\hline August & 101.3 & $\ldots$ & 98.2 & 100.0 & 104.3 & 107.5 & 110.6 & 100.0 & 100.0 & 100.2 \\
\hline September & 104.4 & $\ldots$ & 107.2 & 100.0 & 102.1 & 108.4 & 110.6 & 100.0 & 100.0 & 100.2 \\
\hline October & 104.8 & $\ldots$ & 104.4 & 1112 & 104.3 & 100.9 & 115.1 & 100.0 & 100.0 & 101.1 \\
\hline Novenber & 101.9 & $\ldots$ & 97.6 & 112.5 & 108.1 & 110.8 & 111.8 & 99.0 & 100.0 & 94.8 \\
\hline December & 100.7 & $\ldots$ & 98.8 & 107.8 & 108.1 & 100.9 & 111.5 & 99.0 & 100.0 & 94.5 \\
\hline 1999 & 105.6 & $\ldots$ & 103.1 & 106.7 & 109.9 & 104.8 & 114.3 & 107.7 & 109.0 & 98.4 \\
\hline January & 104.3 & $\cdots$ & 108.5 & 104.1 & 108.1 & 103.7 & 111.0 & 99.0 & $\$ 00.0$ & 96.5 \\
\hline February & 103.7 & $\ldots$ & 104.8 & 107.3 & 108.1 & 106.4 & 112.4 & 99.0 & 100.0 & 96.5 \\
\hline March & 203.4 & $\cdots$ & 103.8 & 107.3 & 108.1 & 106.4 & 112.4 & 99.0 & 100.0 & 96.9 \\
\hline April & 103.7 & $\ldots$ & 104.9 & 107.3 & 108.1 & 106.0 & 112.4 & 99.0 & 100.0 & 96.9 \\
\hline May & 104.3 & 4.27 & 1060 & 107.3 & 108.1 & 106.0 & 113.5 & 99.0 & 100.0 & 98.3 \\
\hline June & 103.2 & 0.61 & 102.8 & 107.3 & 108.1 & 106.0 & 114.1 & 99.0 & 100.0 & 98.3 \\
\hline July & 103.4 & -7.95 & 102.2 & 107.3 & 108.1 & 104.2 & 119.0 & 102.3 & 100.0 & 98.3 \\
\hline Angust & 106.4 & 5.09 & 101.2 & 107.3 & 108.1 & 104.5 & 115.2 & 104.5 & 12.1 .5 & 99.4 \\
\hline September & 108.1 & 3.51 & 100.2 & 107.3 & 112.4 & 105.4 & 115.3 & 119.0 & 121.5 & 99.1 \\
\hline October & $\mathfrak{1 0 8 . 3}$ & 3.43 & 100.0 & 107.3 & 114.5 & 105.9 & 115.1 & 120.6 & 121.5 & 99.0 \\
\hline November & 108.9 & 6.82 & 101.6 & 107.3 & 114.5 & 102.0 & 113.6 & 124.6 & 121.5 & 100.9 \\
\hline December & 108.8 & 8.12 & 101.3 & 103.6 & 112.4 & 101.2 & 117.2 & 127.0 & 121.5 & 100.9 \\
\hline 2000 & 111.1 & 5.30 & 101.7 & 102.6 & 122.7 & 104.8 & 119.1 & 129.0 & 126.2 & 102.9 \\
\hline January & 108.3 & 3.80 & 99.9 & 102.6 & 112.4 & 101.2 & 217.2 & 127.0 & 121.5 & 100.7 \\
\hline February & 108.6 & 4.73 & 100.4 & 102.6 & $1: 2.4$ & 101.4 & 117.6 & 127.0 & 321.5 & 101.7 \\
\hline March & 109.4 & 5.82 & 102.4 & 102.6 & 114.5 & 101.5 & 117.6 & 127.0 & 121.5 & 101.6 \\
\hline April & 109.1 & 5.22 & 100.3 & 102.6 & 114.5 & 103.9 & 116.5 & 127.0 & 121.5 & 103.7 \\
\hline May & 214.0 & 9.37 & 110.5 & 102.6 & 121.0 & 104.2 & 116.5 & 127.0 & 128.5 & 103.7 \\
\hline June & 113.9 & 10.35 & 111.5 & 102.6 & 121.0 & 104.1 & 113.5 & 127.0 & 128.5 & 100.3 \\
\hline July & 111.0 & 7.29 & 100.7 & 102.6 & 121.0 & 106.1 & 118.9 & 127.0 & 128.5 & 102.4 \\
\hline August & 111.2 & 4.44 & 101,3 & 102.6 & 121.0 & 106.1 & 118.9 & 127.0 & 128.5 & 102.4 \\
\hline Seplember & 1.12 .8 & 4.35 & 101.2 & 102.6 & 138.1 & 107.8 & 122.7 & 128.4 & 128.5 & 104.2 \\
\hline October & 111.9 & 3.25 & 99.6 & 102.6 & 132.3 & 107.3 & 122.7 & 128.4 & 128.5 & 104.2 \\
\hline November & 111.3 & 2.20 & 94.6 & 102.6 & 132.1 & 106.8 & 123.6 & 137.8 & 128.5 & 105.2 \\
\hline Decembet & 112.3 & 3.20 & 97.6 & 102.7 & 132,1 & 106.8 & 123.9 & 137.8 & 128.5 & 105.2 \\
\hline \multicolumn{11}{|l|}{2001} \\
\hline January & $11 . .7$ & 3.17 & 96.5 & 102.6 & 131.7 & 106.7 & $\$ 18.6$ & 140.5 & 128.5 & 103.1 \\
\hline February & 111.2 & 2.43 & 94.6 & 102.6 & 131.7 & 103.5 & 117.7 & 144.0 & 130.3 & 103.1 \\
\hline March & 111.9 & 2.27 & 91.4 & 106.7 & 131.7 & 109.0 & 118.6 & 150.1 & 130.3 & 103.1 \\
\hline April & 118.2 & 8.29 & 90.2 & 118.8 & 138.1 & 106.6 & 123.5 & 198.7 & 130.3 & 105.2 \\
\hline May & 118.2 & 3.69 & 90.3 & 118.8 & 138.1 & 106.8 & 123.5 & 198.7 & 130.3 & 105.4 \\
\hline June & 123.9 & 8.78 & 93.2 & 118.8 & 138.1 & 114.6 & 134.1 & 222.1 & 130.3 & 108.3 \\
\hline July & 131.1 & 18.18 & 101.3 & 126.6 & 124.4 & 115.6 & 136.2 & 243.5 & 130.3 & 131.3 \\
\hline August & 133.5 & 20.10 & 103.0 & 133.4 & 142.3 & 117.3 & 137.7 & 244.7 & 130.3 & 131.9 \\
\hline Septcmber & 134.5 & 19.23 & 103.8 & 129.7 & 146.6 & 119.7 & 154.4 & 240.0 & 130.3 & 130.2 \\
\hline October & 135.3 & 20.93 & 104.4 & 137.7 & 139.8 & 115.0 & 161.8 & 245.3 & 130.3 & 131.0 \\
\hline
\end{tabular}

Source: Departrnent of Statistics, Ministry of Planning and Economic Affairs. 
Table 8. Liberia: Central Government Revenue, 1997-2001

(In millions of U.S. dollars)

\begin{tabular}{|c|c|c|c|c|c|}
\hline & 1997 & 1998 & 1999 & 2000 & $\begin{array}{r}2001 \\
\text { Est. }\end{array}$ \\
\hline Tax revenue & 25.2 & 51.6 & 61.7 & 70.9 & 68.7 \\
\hline Taxes on income and profits & 3.1 & 10.5 & 15.0 & 15.6 & 11.1 \\
\hline Corporate and partnership & 0.3 & 8.0 & 7.7 & 4.8 & 4.5 \\
\hline Individual & 0.4 & 1.9 & 5.5 & 9.3 & 5.9 \\
\hline Withholding tax on nouresidents & 2.2 & 0.0 & 0.0 & 0.2 & 0.0 \\
\hline Reconstruction tax & 0.2 & 0.6 & 1.8 & 1.3 & 0.7 \\
\hline Taxes on property & 0.1 & 0.1 & 0.1 & 0.2 & 0.2 \\
\hline Taxes on goods and services & 0.9 & 4.9 & 11.7 & 17.3 & 23.1 \\
\hline Sales tax & 0.0 & 0.0 & 0.0 & 0.0 & 1.2 \\
\hline Excise tax & 0.2 & 0.8 & 0.7 & 0.7 & 0.8 \\
\hline Stumpage fees and land rental & 0.0 & 0.7 & 1.7 & 6.7 & 12.8 \\
\hline Rice stabilization fee & 0.0 & 0.0 & 1.7 & 1.4 & 1.6 \\
\hline Motor vehicle taxes & 0.1 & 0.5 & 1.5 & 1.3 & 1.2 \\
\hline Petroleum sales tax & 0.6 & 2.9 & 6.1 & 7.2 & 5.5 \\
\hline Maritime revenue & 16.7 & 17.3 & 15.2 & 14.5 & 16.0 \\
\hline Taxes on international trade & 4.4 & 18.8 & 19.7 & 23.3 & 18.3 \\
\hline Taxes on imports & 3.6 & 18.4 & 19.3 & 23.0 & 18.1 \\
\hline Taxes on exports & 0.8 & 0.4 & 0.4 & 0.3 & 0.1 \\
\hline Other taxes & 0.2 & 0.0 & 0.0 & 0.0 & 0.0 \\
\hline Nontax revenue & 0.4 & 2.2 & 3.8 & 4.6 & 5.3 \\
\hline Total revenue & 25.6 & 53.8 & 65.5 & 75.5 & 74.0 \\
\hline Grants & $\cdots$ & 5.5 & 8.3 & 5.3 & 6.7 \\
\hline Total revenue and grants & $\cdots$ & 59.3 & 73.8 & 80.8 & 80.7 \\
\hline
\end{tabular}

Sources: Liberian authorities; and Fund staff estimates. 
Table 9. Liberia: Economic Classification of Central Government Expenditure, 1997-2001

(In millions of U.S. dollars)

\begin{tabular}{lrrrrr}
\hline & 1997 & 1998 & 1999 & 2000 & $\begin{array}{r}2001 \\
\text { Est. }\end{array}$ \\
\hline Current expenditure & 20.9 & 36.9 & 40.5 & 79.6 & 55.7 \\
Wages and salaries & 4.7 & 6.1 & 10.5 & 17.7 & 20.4 \\
Other goods and services & 12.2 & 22.7 & 17.0 & 36.9 & 30.8 \\
Subsidies and grants & 3.2 & 4.0 & 9.2 & 15.5 & 1.2 \\
Debt service & 0.9 & 4.1 & 3.8 & 9.6 & 3.3 \\
Capital expenditure & & & & & \\
Internally financed & 0.9 & 12.9 & 18.9 & 7.8 & 17.4 \\
Externally financed & $\ldots$ & 7.4 & 12.5 & 1.4 & 10.7 \\
& $\ldots$ & 5.5 & 6.4 & 6.3 & 6.7 \\
Unallocable & & & & & \\
& 6.4 & 8.2 & 4.0 & 1.0 & 0.0 \\
Total & & & & & \\
& 28.2 & 58.0 & 63.5 & 88.4 & 73.1 \\
\hline
\end{tabular}

Sources: Liberian authorities; and Fund staff estimates. 
Table 10. Liberia: Summary Accounts of the Central Bank of Liberia, December 1998-October 2001 (In millions of Liberian dollars; unless otherwise indicated; end of period)

\begin{tabular}{|c|c|c|c|c|}
\hline & 1998 & 1999 & 2000 & 2001 \\
\hline & Dec. & Dec. & Dec. & Oct. \\
\hline Net foreign assets & $28,655.9$ & $25,910.1$ & $27,254.3$ & $31,624.6$ \\
\hline Foreign assets & 26.7 & $\quad 17.0$ & 11.4 & 16.3 \\
\hline Foreign liabilities & $-28,629.2$ & $-25,893.0$ & $-27,242.9$ & $-31,608.3$ \\
\hline Net domestic assets & $-27,717.5$ & $-24,930.2$ & $-25,869.6$ & $-30,072.8$ \\
\hline Domestic credit & $35,861.5$ & $32,709.6$ & $36,769.2$ & $43,556.7$ \\
\hline Claims on the government (net) & $35,824.4$ & $32,676.1$ & $36,643.0$ & $43,365.7$ \\
\hline Claims & $35,687.0$ & $32,605.1$ & $36,643.0$ & $43,339.8$ \\
\hline \multicolumn{5}{|l|}{ Contra-entry for use of Fund credit } \\
\hline and overdue charges & $28,159.2$ & $25,479.2$ & $26,824.8$ & $31,259.4$ \\
\hline Other & $7,527.8$ & $7,125.9$ & $9,818.2$ & $12,080.3$ \\
\hline Deposits & -137.4 & -71.0 & 0.0 & -25.9 \\
\hline Claims on private sector & 25.5 & 24.6 & 48.9 & 12.4 \\
\hline Claims on public corporations & 5.8 & 5.5 & 0.0 & 0.0 \\
\hline Claims on nonbank financial institutions & 1.5 & 1.5 & 0.0 & 0.0 \\
\hline Claims on domestic banks & 4.3 & 1.7 & 77.3 & 178.7 \\
\hline Other iterns (net) & $-63,579.1$ & $-57,639.7$ & $-62,638.8$ & $-73,629.5$ \\
\hline Of which: capital account & $-9,259.2$ & $-7,735.2$ & $-7,675.1$ & $-8,085.8$ \\
\hline Reserve money & 938.4 & 979.9 & $1,384.7$ & $1,551.8$ \\
\hline Currency in circulation & 647.0 & 647.4 & 821.1 & 876.5 \\
\hline Bank deposits at Central Bank of Liberia & 291.4 & 274.1 . & 488.0 & 600.5 \\
\hline Other deposits & 0.0 & 58.4 & 75.6 & 74.8 \\
\hline \multicolumn{5}{|l|}{ Memorandum items: } \\
\hline Required reserves & 295.1 & 346.0 & 283.9 & 389.2 \\
\hline (reserve requirement (Liberian dollar deposits, in percent & 22.0 & 22.0 & 22.0 & 50.0 \\
\hline (reserve requirement (U.S. dollar deposits, in percent)) & 22.0 & 22.0 & 22.0 & 18.0 \\
\hline Excess reserves & -3.7 & -71.9 & 204.1 & 211.3 \\
\hline
\end{tabular}

Sources: Liberian authorities; and Fund staff estimates. 
Table 11. Liberia: Summary Accounts of Deposit Money Banks, December 1998 - October 2001 (In millions of Liberian dollars; unless otherwise indicated; end of period)

\begin{tabular}{|c|c|c|c|c|}
\hline & 1998 & 1999 & 2000 & 2001 \\
\hline & Dec. & Dec. & Dec. & Oct. \\
\hline Net foreign assets & 554.8 & 660.2 & 957.7 & $1,208.2$ \\
\hline Foreign assets & 447.9 & 660.2 & 529.8 & 652.8 \\
\hline Foreign liabilities & -106.9 & 0.0 & -427.9 & -555.4 \\
\hline Net domestic assets & 17.9 & 97.2 & -841.9 & $-1,091.0$ \\
\hline Domestic Credit & $1,578.5$ & $1,468.9$ & $1,385.6$ & $1,783.2$ \\
\hline Claims on governement (net) & 418.2 & 523.5 & 559.4 & 687.2 \\
\hline Claims & 306.2 & 358.1 & 425.3 & 562.6 \\
\hline Deposits & -112.0 & -165.4 & -134.1 & -124.6 \\
\hline In U.S. dollars & $\ldots$ & $\ldots$ & .72 .7 & -83.5 \\
\hline In Liberian dollars & $\ldots$ & $\ldots$ & -206.8 & -208.0 \\
\hline Claims on private sector & $1,148.1$ & 900.9 & 663.2 & 834.5 \\
\hline Claims on public enterprises & 12.2 & 44.5 & 39.5 & 85.7 \\
\hline Claims on Nonbank financial institutions & 0.0 & 0.0 & 123.6 & 175.9 \\
\hline Other items (net) & $-1,560.6$ & $-1,371.7$ & $-2,227.5$ & $-2,874.3$ \\
\hline Of which: capital account & 36.3 & 685.6 & 889.9 & $1,221.2$ \\
\hline Reserves & 768.5 & 815.2 & $1,174.5$ & $1,445.7$ \\
\hline Total deposit liabilities & $1,341.3$ & $1,572.6$ & $1,290.3$ & $1,562.9$ \\
\hline Demand deposits & $1,005.5$ & $1,247.9$ & 898.8 & $1,084.2$ \\
\hline In U.S. dollars & $\ldots$ & $\quad \ldots$ & 804.5 & 901.6 \\
\hline In Liberian dollars & ... & $\ldots$ & 94.3 & 182.6 \\
\hline Time and savings deposits $1 /$ & 335.8 & 324.7 & 391.5 & 478.6 \\
\hline In U.S. dollars & $\ldots$ & $\ldots$ & 266.8 & 314.7 \\
\hline In Liberian dollars & $\cdots$ & $\ldots$ & 122.3 & 154.4 \\
\hline \multicolumn{5}{|l|}{ Memorandum items: } \\
\hline U.S. dollar deposits (valued in Liberian dollars) & $\ldots$ & $\ldots$ & $1,144.0$ & $1,299.8$ \\
\hline (as a percent of total deposits) & $\ldots$ & $\ldots$ & 98.9 & 90.4 \\
\hline
\end{tabular}

Sources: Liberian authorities; and Fund staff estimates.

1/ Exludes accrued interest. 
Table 12. Liberia: Major Exports, 1987-88 and 1999-2001 1/

$\begin{array}{lllll}1987 & 1988 & 1999 & 2000 & 2001\end{array}$

Est.

\begin{tabular}{|c|c|c|c|c|c|}
\hline \multirow[b]{2}{*}{ Total exports } & \multicolumn{5}{|c|}{ (In millions of U.S. dollars) } \\
\hline & 401.2 & 442.8 & 58.9 & 120.3 & 127.4 \\
\hline Rubber & 94.2 & 106.5 & 33.1 & 57.1 & 67.4 \\
\hline Timber & 61.3 & 93.8 & 23.4 & 61.0 & 58.9 \\
\hline Cocoa & 6.0 & 6.3 & 1.3 & 0.6 & 0.5 \\
\hline Coffee & 10.0 & 5.7 & 0.7 & 0.5 & 0.0 \\
\hline Iron ore & 206.4 & 208.1 & 0.0 & 0.0 & 0.0 \\
\hline \multirow[t]{2}{*}{ Other } & 23.3 & 22.4 & 0.4 & 1.1 & 0.6 \\
\hline & \multicolumn{5}{|c|}{ (Annual percentage changes) } \\
\hline Total exports & 5.2 & 10.4 & 37.6 & 104.3 & 5.9 \\
\hline Rubber & 11.9 & 13.1 & 16.0 & 72.6 & 18.1 \\
\hline Timber & 85.2 & 53.0 & 90.6 & 160.3 & -3.4 \\
\hline Cocoa & -32.6 & 5.0 & -20.8 & -51.8 & -11.5 \\
\hline Coffee & -38.3 & -43.0 & 196.5 & -28.1 & -94.7 \\
\hline Iron ore & -5.2 & 0.8 & $\ldots$ & $\ldots$ & $\ldots$ \\
\hline \multirow[t]{2}{*}{ Other } & $\ldots$ & -3.9 & 188.7 & 180.7 & -49.1 \\
\hline & \multicolumn{5}{|c|}{ (In percent of total) } \\
\hline Total exports & 100.0 & 100.0 & 100.0 & 100.0 & 100.0 \\
\hline Rubber & 23.5 & 24.1 & 56.2 & 47.4 & 52.9 \\
\hline Timber & 15.3 & 21.2 & 39.8 & 50.7 & 46.2 \\
\hline Cocoa & 1.5 & 1.4 & 2.2 & 0.5 & 0.4 \\
\hline Coffee & 2.5 & 1.3 & 1.2 & 0.4 & 0.0 \\
\hline Iron ore & 51.4 & 47.0 & 0.0 & 0.0 & 0.0 \\
\hline Other & 5.8 & 5.1 & 0.7 & 0.9 & 0.4 \\
\hline
\end{tabular}

Sources: Liberian authorities; and Fund staff estimates.

1/ In U.S. doliar values. 
Table 13: Liberia, Merchandise Trade- Imports by Category, 1987-88 and 1999-2001

\begin{tabular}{|c|c|c|c|c|c|}
\hline & 1987 & 1988 & 1999 & 2000 & $\begin{array}{r}2001 \\
\text { Est. }\end{array}$ \\
\hline & \multicolumn{5}{|c|}{ (In millions of U.S. dollars) } \\
\hline Total & 307.6 & 331.3 & 209.6 & 189.3 & 180.9 \\
\hline Food and live animals & 58.9 & 58.4 & 54.0 & 53.9 & 56.3 \\
\hline of which: rice & 27.3 & 44.7 & 21.8 & 26.2 & 30.7 \\
\hline Beverages and tobacco & 4.5 & 4.8 & 7.7 & 6.3 & 5.8 \\
\hline Crude materials inedible excluding fuel & 2.4 & 2.9 & 11.3 & 6.6 & 3.8 \\
\hline Mineral fuels, lubricants & 69.7 & 69.4 & 50.7 & 44.9 & 37.2 \\
\hline of which: petroleurn & 59.7 & 59.4 & 47.3 & 42.9 & 35.2 \\
\hline Animal, vegetable oil & 4.1 & 2.2 & 3.6 & 3.3 & 2.3 \\
\hline Chemicals and related products & 22.1 & 18.9 & 14.8 & 14.3 & 8.4 \\
\hline Manufactured goods & 55.1 & 59.0 & 17.0 & 20.8 & 19.4 \\
\hline Machinery and transport equipment & 73.5 & 94.4 & 38.9 & 30.0 & 31.9 \\
\hline Miscellaneous manufactured & 14.2 & 18.9 & 11.5 & 9.3 & 4.5 \\
\hline \multirow[t]{2}{*}{ Commodities and transactions not elsewhere specified } & 3.1 & 2.4 & 0.0 & 0.0 & 11.4 \\
\hline & \multicolumn{5}{|c|}{ (Annual percent change) } \\
\hline Total & 18.8 & 7.7 & 30.0 & -9.7 & -4.4 \\
\hline Food and live animals & 9.9 & -0.8 & 10.9 & -0.2 & 4.5 \\
\hline of which: rice & -49.4 & 63.9 & -27.2 & 20.0 & 17.2 \\
\hline Beverages and tobacco & 12.5 & 6.7 & 5.7 & -18.8 & -8.3 \\
\hline Crude materials inedible excluding fuel & -31.4 & 20.8 & 146.8 & -41.8 & -41.5 \\
\hline Mineral fuels, lubricants & 31.8 & -0.4 & 28.8 & -11.5 & -17.1 \\
\hline of which: petroleum & 39.2 & .0 .5 & 30.8 & -9.4 & -17.9 \\
\hline Animal, vegetable oil & 28.1 & -46.3 & 210.5 & -7.8 & -30.7 \\
\hline Chemicals and related products & -16.0 & -14.5 & 63.5 & -3.4 & -41.6 \\
\hline Manufactured goods & 52.6 & 7.1 & 13.2 & 22.0 & -6.6 \\
\hline Machinery and transport equipment & 24.6 & 28.4 & 40.4 & -23.0 & 6.3 \\
\hline Miscellaneous manufactured & -22.4 & 33.1 & 83.0 & -19.0 & -51.9 \\
\hline \multirow[t]{2}{*}{ Commodities and transactions not elsewhere specified } & 47.6 & -22.6 & 0.0 & 0.0 & $\ldots$ \\
\hline & \multicolumn{5}{|c|}{ (In percent of total) } \\
\hline Total & 100.0 & 100.0 & 100.0 & 100.0 & 100.0 \\
\hline Food and live animals & 19.1 & 17.6 & 25.8 & 28.5 & 31.1 \\
\hline of which: rice & 8.9 & 13.5 & 10.4 & 13.8 & 17.0 \\
\hline Beverages and tobacco & 1.5 & 1.4 & 3.7 & 3.3 & 3.2 \\
\hline Crude materials inedible excluding fuel & 0.8 & 0.9 & 5.4 & 3.5 & 2.1 \\
\hline Mineral fuels, lubricants & 22.7 & 20.9 & 24.2 & 23.7 & 20.6 \\
\hline of which: petroleum & 19.4 & 17.9 & 22.6 & 22.6 & 19.4 \\
\hline Animal, vegetable oil & 1.3 & 0.7 & 1.7 & 1.8 & 1.3 \\
\hline Chemicals and related products & 7.2 & 5.7 & 7.1 & 7.6 & 4.6 \\
\hline Manufactured goods & 17.9 & 17.8 & 8.1 & 11.0 & 10.7 \\
\hline Machinery and transport equipment & 23.9 & 28.5 & 18.6 & 15.8 & 17.6 \\
\hline Miscellaneous manufactured & 4.6 & 5.7 & 5.5 & 4.9 & 2.5 \\
\hline Commodities and transactions not elsewhere specified & 1.0 & 0.7 & 0.0 & 0.0 & 6.3 \\
\hline
\end{tabular}


Table 14: Direction of Exports, 1989, and 2000-2001

(Percent of total)

\begin{tabular}{|c|c|c|c|}
\hline Country & 1989 & 2000 & 2001 \\
\hline United States & 17.2 & 5.2 & 9.4 \\
\hline Europe & 65.2 & 75.3 & 59.5 \\
\hline Belgium & $\ldots$ & 47.1 & 4.8 \\
\hline Germany & 25.0 & 0.5 & 10.5 \\
\hline Italy & 14.6 & 9.4 & 3.8 \\
\hline France & 7.7 & 3.9 & 7.5 \\
\hline Norway & 2.3 & 3.5 & 23.8 \\
\hline Switzerland & 0.0 & 5.6 & 0.5 \\
\hline Turkey & 2.0 & 1.5 & 3.1 \\
\hline Spain & 3.1 & 2.7 & 3.4 \\
\hline Portugal & 0.3 & 0.4 & 0.4 \\
\hline Netherlands & 2.4 & 0.2 & 0.9 \\
\hline United Kingdom & 1.5 & 0.4 & 0.7 \\
\hline Southeast Asia & 4.4 & 12.3 & 20.5 \\
\hline Korea, Republic of & 0.4 & 4.2 & 2.0 \\
\hline Singapore & 2.5 & 3.1 & 6.5 \\
\hline Malaysia & 0.0 & 1.0 & 1.4 \\
\hline Bangladesh & 0.0 & 0.8 & 2.0 \\
\hline Pakistan & 1.4 & 2.4 & 0.0 \\
\hline ECOWAS 1/ & 1.0 & 0.9 & 4.1 \\
\hline Other & 12.2 & 6.4 & 6.5 \\
\hline Total & 100.0 & 100.0 & 100.0 \\
\hline
\end{tabular}

Sources: IMF, Direction of Trade Statistics.

1/ Economic Community of West African States. 
Table 15. Liberia: Summary of Tax System

(As of December 31, 2001)

\begin{tabular}{cccc}
\hline $\operatorname{Tax}$ & Nature of Tax & Exemptions and Deductions \\
\hline
\end{tabular}

A. Central govenment

Taxes on income and profits

1.1 Taxes on companies

1.11 Taxes on company income

Annual tax on net profits received by

Liberian and foreign companies. Liberian companies are taxed on income derived from their operations within Liberia. Companies with tax liability of $\$ 500$ or more are required to make payments on a current payment basis.
1.12 Tax on partnerstip income

1.12 Turnover tax
Registered charities are exempted from income and profit taxes

Income derived from sources outside Liberia is exempt, if the majority of the voting power of a domestic company is held by foreigners or nonresidents. Also exernpt are earnings from the operation of vessels if not derived exclusively from coastwide operation by

resident corporations. Under the Investment

Code, companies with qualifying Liberian

personnel are exempted from all income taxes

for a period of five years or until their

accumulated profits exceed 150 percent of

their initial investment. This tax holiday may

be extended to ten years in certain cases.

Donations to approved organizations are

deductible, subject to a limit of 15 percent of

net income.

Partnerships are granted the same deductions on corporations. No deductions may be made for personal or family expenses of any

partner. exceeds $\$ 500$, payments must be made on current payment basis.

Payable in lieu of income tax by partnerships and companies with a gross income not exceeding LS 5,000,000. For partnerships and companies with gross income higher than $L \$ 5,000,000$, the turnover tax is creditable against income
35 percent.
35 percent. Creditable against income tax of individual partners.

$4 \%$ of gross income. 
1.2 Taxes on individuals

1.21 Income tax

1.22 National

reconstruction tax

(suspended as of July 1, 2001)

1.23 Turnover tax

1.23 Petty trader tax
The tax is payable annually by all citizens and residents of Liberia on net income received from all sources, including capital gains. Partners must file individual returns of distributive income of all partnership(s) together with income from other sources. Employees' taxes are withheld from salaries or wages (for wages and salaries over $\$ 84$ a month) and self-employed individuals with tax liability of $\$ 500$ or more are required to make payments on a current payment basis. There are established estimates for taxpayers who do not keep records of their income.

$$
\text { , }
$$

The tax is levied on every self-employed, salaried, or wage-earning citizen and foreign resident in Liberia over 16 years of age on gross income for each month of employment or fraction thereof.

Payable in lieu of income tax by individuals with a gross income not exceeding IS 5,000,000. For individuals with gross income higher than $\mathrm{LS}$ $5,000,000$, the turnover tax is creditable against incone tax.

Payable in tieu of income tax and turnover tax by individuals with gross income of less than L\$200,000
Proceeds of life and health insurance, as well as sickness, disability, and death benefits,

gifts, bequests, and interest on certain

government obligations are excluded from the income of the recipient.

Noncash benefits up to L\$ 100,000 are excluded from the taxable income.

Donations to approved organizations are deductible within the limit of 15 percent of net income.

Medical expenses and insurance premiums up to a specified limit.

Partnership taxes and, where applicable, turnover taxes paid by an individual are credited against the income tax due.

\section{Income (in LS) \\ Up to $\$ 12,000$ \\ 12,001 to $50,000 \quad 240$ plus $5 \%$ of \\ Rate \\ 50,001 to $100,000 \quad$ excess over 12,000 2,140 plus $10 \%$ of excess over 50,000 100,001 to $200,000 \quad 7,140$ plus $15 \%$ of
excess over 100,000 200,001 to $400,000 \quad 22,140$ plus $20 \%$ of excess over 200,000 $\begin{array}{ll}400,001 \text { to } 800,000 & 62,140 \text { plus } 25 \% \text { of } \\ \text { excess over } 400,000\end{array}$ 800,001 to $1200,000 \quad 162,140$ plus $30 \%$ of excess over 800,000 1200,001 and over 282,140 plus $35 \%$ of excess over 1200,000}

Payable on monthly income as follows:

$\begin{array}{lc}\text { (In dollars) } & \text { (In percent) } \\ 1 \text { to } 200 & 1.0 \\ 201 \text { to } 500 & 4.5 \\ 501 \text { to } 1,000 & 7.5 \\ 1,001 \text { and above } & 8.0\end{array}$

1,001 and above $\quad 8.0$

$4 \%$ of turnover

L $\$ 400$ for traders using a portable business structure,

L $\$ 1,000$ for traders using a fixed open structure with roof,

L $\$ 2,000$ for traders using a fixed structure,

L\$ 200 for traders falling under the categories above, who do business exclusively outside Montserraco county. 


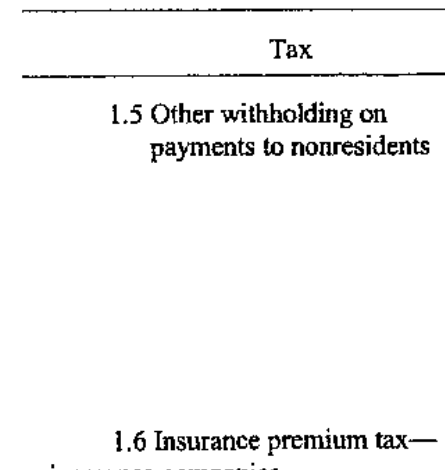
insurance companits

2. Payroll taxes

3. Taxes on property

3.1 Real estate tax

3.11 Real estate tax

3.12 Wiltholding tax on rental income

3.2 Net wealth taxes

3.3 Death and gitt taxes

Nature of Tax

Exemptions and Deductions

Rates

Levied on the gross amount of interest,

dividends, royalties, rents, compensations,

and other fixed or determinable incomes

earned in Liberia by nonresident

foreigners. Under certain conditions,

taxpayers may be gronted appropriate relief

at the discretion of the minister in cases of

inequity in tax burden, for example, double

taxation.

Annual tax on all gross premium received

from policy by insurer on direct risks

written in Liberia and also if a domestic

insurer on direct risks is situated in any

other country or countries.

None.

Exemption from taxation are:

(a) interest: earned on deposits with banks in

Liberia;

(b) interests, commissions, and other charges

levied on short-term commercial transaclions; and

(c) items of income specifically listed in

concession agreements
4 percent.
Levied on land located in a city, township or commonwealth district and the assessed value of any improvement thereon.

value of any improvement thereon.
Valuation of the property is determined by the tax administration.

xemption from taxation are:

(a) government properties;

(b) properties of churches or other religious societies held for educational, charitable, or fraternal purposes;

(c) buildings and other properties owned by

the University of Liberia; and

(d) properties held by foreign governments

not owned by private individuals.

Annual tax on rent or lease payments of realty. The tax is withheld by the leasor at the time of rent payment.

None.

None.
5 percent on unimproved and in owns and cities, and 5 cents per acre on rural land, including farmland, with a minimum of

$\$ 5,000.2$ percent on improved land in commercial use. l percent on improved land for industrial use. $1 / 2$ of 1 percent on residential property.

10 percent of the rent or lease. Creditable against income tax. 


\begin{tabular}{cccc}
\hline $\operatorname{Tax}$ & Nature of Tax & Exemptions and Deductions \\
\hline 3.4 Property transfer taxes & None. &
\end{tabular}

4. Taxes on domestic goods and services

4.1 Goods and Services tax

4.1.1 Goods tax

(in force since July 1,2001 )

4.1.2 Services tax

(in force since July 1,2001 )
Ad valorem tax paid by the manufacturer or the importer. For local manufacturers, the taxable amount is the ex-factory price of the respective goods. For importers, the taxable amount includes the $\mathrm{CF}$ value,

import-related services, and import duties. Payable monthly.

Add valorem tax on taxable services,

including electricity services,

telecommunications services, the provision

of water for a fee, holel accomodation,

meals in restaurants, gambling services,

sale of tickets by intemational transport

services (air, sea, and land) services of a

travel agency, and sporting services

(including ticket sales). Payable monthly.
The goods tax on locally produced cement, imported rice, and petroleum products has been suspended until Dec 31, 2001, and is expected to remain suspended for the near future.

Exempt goods include

(1) Goods destined for export,

(2) capital goods and raw materials or other inputs for use directly in manufacturing

(3) goods incidental to a supply of taxabic services

(4) food products purchased by educational and philantropic institutions,

(5) various medical and educalional goods, as specified by regulation by the Minister of Finance, and

(6) supply of goods for the relief of distressed persons in the case of natural disasters or other humanitarian emergencies. 
$\operatorname{Tax}$

4.2 Excise taxes

4.3 Taxes on timber production

\subsection{Rubber sales tax}

(suspended as of July 1, 2001)
Nature of Tax

Ad valorem tax paid by the importer on the

CIF price plus import taxes, or by the

manufacturer on the cost price value.

Taxes on the production of logs, lumber. planks, and other partly manufactured timber products, and a land rental fee for concession holders. permissible under certain circumstance
The excise tax on petroleum products has been suspended until Dec 31, 2001, and is expected to remain suspended for the near future.

\section{Rates}

Rate(In percent)

(1) Stones and asbestos 30.0

(2) Metallic ores and coal products $\quad 7.0$

(3) Petroleun products $\quad 7.0$

(4) Rubber and latex 20.0

(5) Roofing and building materials 30.0

(6) Dianonds and precious metals 20.0

(7) Alcoolic beverages (imported) $\quad 15.0$

(8) Alcoolic beverages (local) $\quad 5.0$

$\begin{array}{lr}\text { (10) Tobacco products } & 11.0\end{array}$

$\begin{array}{ll}\text { (10) Tobacco products } & 11.0 \\ \text { (1) Gambling equipment } & 30.0\end{array}$

$\begin{array}{lr}\text { (11) Gambling equipment } & 30.0 \\ \text { (12) Cosmetic products } & 7.0\end{array}$

(13) Luxury goods (preserved fruits, jams,

leather products, jewellery, electric household appliances, motor cars exceeding $2,500 \mathrm{cc}$, cameras, and others) $\quad 20.0$

$\begin{array}{ll}\text { (14) Monosodium ghtamate } & 30.0\end{array}$

(15) Revolvers, pistols, ammunition 50.0 where reforestation is undertaken.

(a) Severance fee U.S.\$1.50 per cu. meter.

(b) Industrial incentive fee on all unprocessed round logs for export varies according to species (from U.S.S 1.44 to 58.56 per cu. meter).

(c) Forest products fee for sawn timber from U.S. $\$ 0.40$ to 30.60 per cu. meter.

(d) Reforestation fee: U.S,\$3.00 per cu. meter (e) Conservation fee: U.S.\$ $1.50-2.50$ per cu. meter

(f) Forest research tax: U.S.S 1.00 per cu. meter

(g) Land rental fee: U.S $\$ 0.50$ per acre

\section{Taxes on intemational trade}

5.1 Taxes on imports 


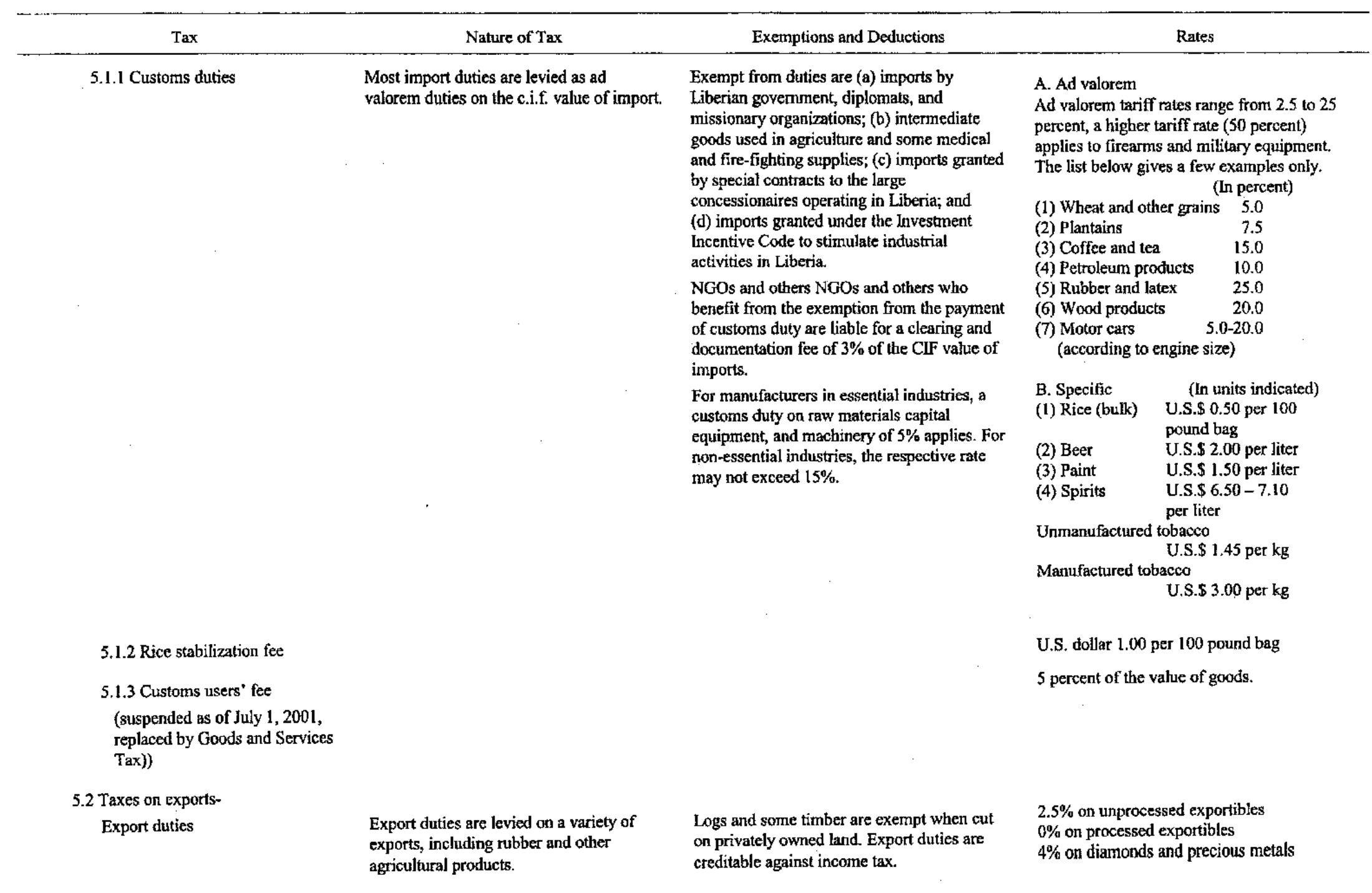

6. Other taxes 


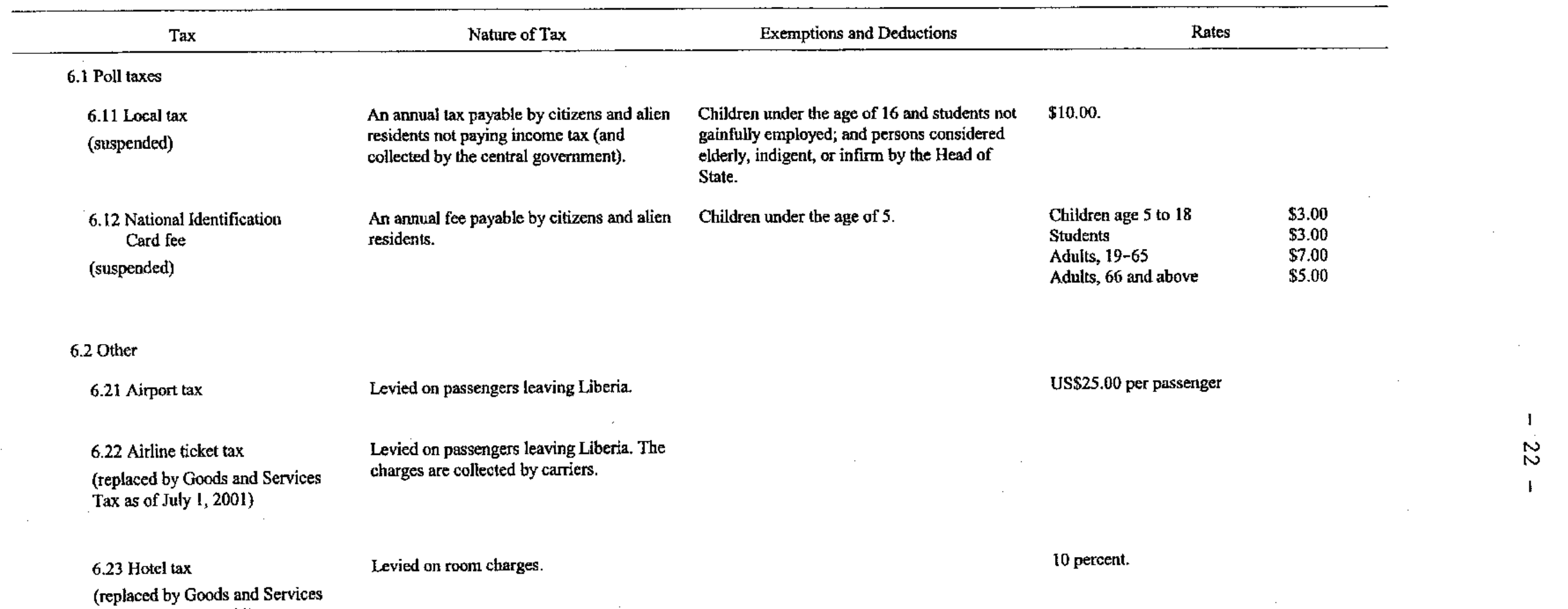

Source: Liberian authorities. 